\title{
Dosage Compensation Systems
}

\section{Contents}

4.1 Introduction: Evolution of Chromosome-Wide Dosage Compensation - 68

4.1.1 Consequences of Gene Dosage Differences Arising from Sex Chromosome Erosion - 70

4.2 The Dosage Compensation Complex of the Fruit Fly Drosophila melanogaster - 71

4.3 X Chromosome Inactivation in Mammals - 75

4.3.1 The Mammalian Dosage Compensation Mechanism - 76

4.3.2 Regulation of XCl in Different Mammals - 82

4.4 X Chromosome Dosage Compensation in Caenorhabditis elegans - 85

References -88 


\section{What You Will Learn in this Chapter}

This chapter provides an introduction to chromosome-wide dosage compensation systems. We will examine the evolution of dosage compensation, which is thought to be driven by the appearance of differentiated sex chromosomes. In a subset of species with X chromosomal sex determination or XY sex chromosome systems, expression of X-linked genes is regulated by chromosome-wide modifications that equalize gene expression differences between males and females. The molecular mechanisms of $\mathrm{X}$ chromosome-wide dosage compensation have been studied in flies, worms, and mammals. Each of these species uses a distinct dosage compensation strategy with a different molecular mechanism. In the worm Caenorhabditis elegans, gene expression on the two X chromosomes of hermaphrodites is reduced to a level that approximates a single $\mathrm{X}$ chromosome in males. The fruit fly Drosophila melanogaster achieves dosage compensation by increased transcription of the single $\mathrm{X}$ chromosome in males to a level that is similar to the two $\mathrm{X}$ chromosomes in females. Lastly, in mammals, one of the two X chromosomes in female cells is transcriptionally inactive and a single $\mathrm{X}$ chromosome is transcribed in both sexes. Studies of dosage compensation systems provide insights into how epigenetic regulation controls gene expression and chromatin organization differentially within a cell.

\subsection{Introduction: Evolution of Chromosome-Wide Dosage Compensation}

Sexual reproduction can be observed in nearly all animal phyla. Different sex determination strategies have evolved, ranging from determination by environmental conditions including temperature to genetic sex determination with specific sex determination genes (Bachtrog et al. 2014). Sex determination systems allow the development of dimorphic phenotypes of males and females in a species. For this chapter, chromosomal sex determination is of primary interest as structural differences of sex chromosomes provide the context for the evolution of dosage compensation systems. In birds, males carry two $\mathrm{Z}$ chromosomes and females possess heteromorphic $\mathrm{Z}$ and $\mathrm{W}$ chromosomes. In mammals, males have an XY and females an XX sex chromosome constitution (Graves 2015). A wide range of XY, XO, and ZW sex chromosome systems have been documented in reptiles, amphibians, fish, and insects (Kaiser and Bachtrog 2010). The gene content of the sex chromosomes is different, though. For example, the snake and bird ZW systems are different and, hence, have evolved independently. Sex chromosomes in one species can correspond to autosomes in a different species, suggesting that sex chromosomes originate through modification of autosome pairs. How autosomes are selected to become sex chromosomes is just beginning to be understood. Evolution acts on sex chromosomes in a variety of ways that are species-, chromosome-, and context-dependent (Bellott et al. 2010). The general idea is that a sex determining locus arises on an ancestral autosome forming a proto-sex chromosome. Subsequent loss of sequences (erosion) on the proto-sex chromosome converts the ancestral autosome pair into a dimorphic sex chromosome pair (• Fig. 4.1).

The erosion of sex chromosomes has been explained as a consequence of recombination suppression. This could likely be facilitated by large inversions in the vicinity of the sex-determining region. Suppressed recombination facilitates the genetic 


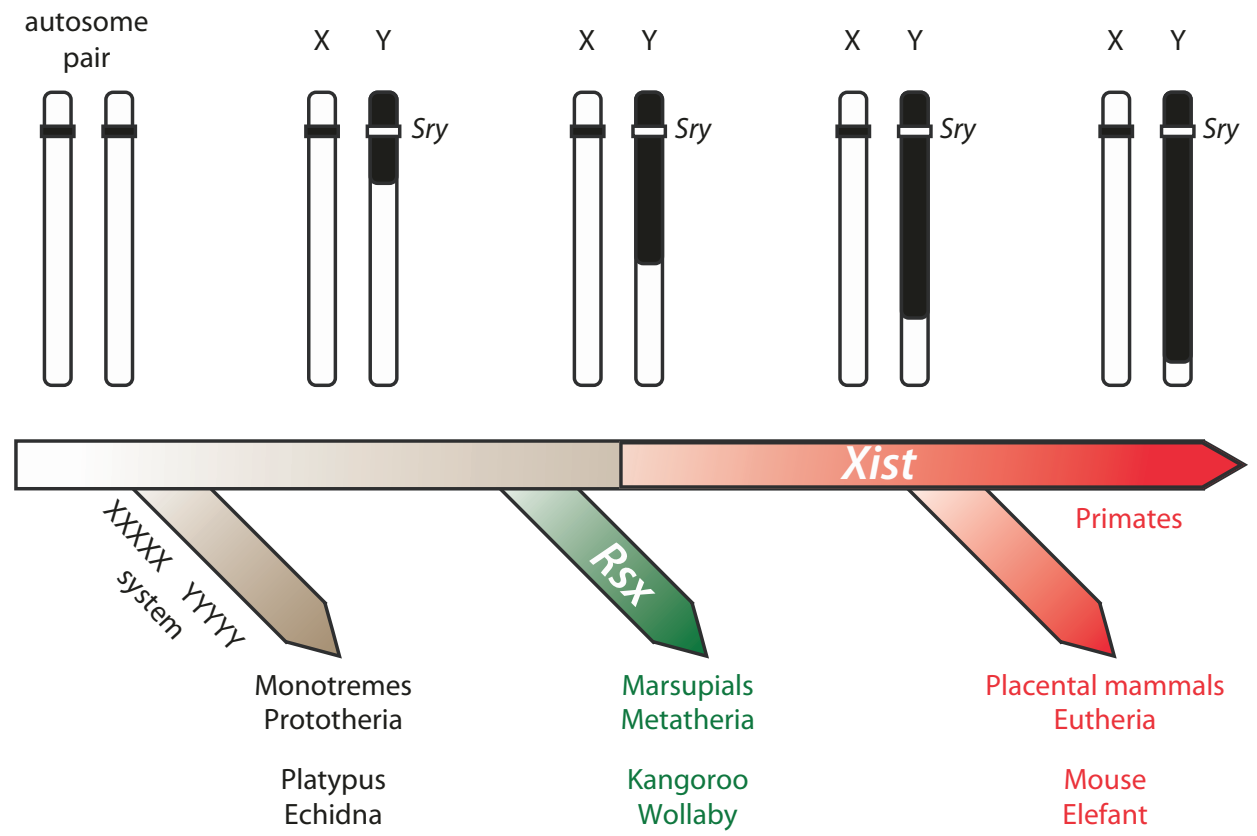

- Fig. 4.1 Evolution of sex chromosomes in mammals. Placental and marsupial mammals possess $\mathrm{XY}$ sex chromosomes that have arisen from an ancestral autosome pair about 210 mya. These sex chromosomes correspond to autosomes in monotremes that possess a system of $5 \mathrm{X}$ and $5 \mathrm{Y}$ chromosomes. Evolution has shaped sex chromosomes by erosion and the addition of autosomal fragments to the sex chromosomes, leading to divergent chromosome structures between marsupials and placental mammals but also within placental mammals. Sequences lost due to erosion are marked in black along the evolving $\mathrm{Y}$ chromosome. As a consequence of the erosion of the Y chromosome, dosage compensation systems have arisen. Although placental and marsupial mammals inactivate the second $\mathrm{X}$ chromosome in female cells, the molecular details of the underlying mechanisms differ. In placental mammals, the Xist gene initiates $\mathrm{X}$ chromosome inactivation. In marsupials, the $R s x$ gene has emerged as the trigger by convergent evolution

linkage between the sex-determining gene and genes that are beneficial for one sex but detrimental to the other. For example, genes that are required for testis development reside on the mammalian $\mathrm{Y}$ chromosome together with the sex determining region $Y(S R Y)$ gene, which specifies male sex. Since females do not inherit a Y chromosome, there are no detrimental consequences from Y-linked genes to them. The $\mathrm{XY}$ sex chromosome system of placental mammals including humans is estimated to have evolved about 210 million years ago (mya) (Marshall Graves 2008). The mouse Y chromosome has lost all but a few genes that act in determining male sex and testis development whereas in other mammals, repeated addition of autosomal fragments to the XY chromosome pair has generated different and larger Y chromosomes. The evolution of different clades of mammals has led to divergent sex chromosome structures. Placental and marsupial mammals possess XY chromosomes that originate from the same ancestral autosome pair. The more distantly related monotremes are examples of species with multiple sex chromosomes (• Fig. 4.1). Platypus and several species of echidna possess $5 \mathrm{X}$ and $5 \mathrm{Y}$ chromosomes, which are not homologous to the XY chromosomes in placental and marsupial mammals but show limited 
homology to the $\mathrm{Z}$ chromosome of birds (Graves 2015; Veyrunes et al. 2008). The human $\mathrm{X}$ chromosome is homologous to an autosomal region in monotremes. Further information on the evolution of mammalian sex chromosome systems can be found in a specialized review (Marshall Graves 2008).

\subsubsection{Consequences of Gene Dosage Differences Arising from Sex Chromosome Erosion}

Loss of genes on the sex-limited chromosome (the $\mathrm{Y}$ in mammals) and the evolution of dimorphic sex chromosomes can have two consequences. Firstly, a divergence from the ancestral gene dosage through erosion of the proto-sex chromosome relative to the ancestral autosomes is incurred. Whether an associated reduction of fitness $^{1}$ is tolerated or can be compensated for, remains to be resolved for each particular system. Secondly, a gene dosage difference between the two sexes arises as one of the sex chromosomes progressively shrinks in size and its gene content diminishes. It is likely that the process of erosion is constrained by deleterious effects caused by these gene dosage changes. Therefore, not all autosomes might be suitable for forming small sex chromosomes. In some instances, sex chromosomes appear to avoid erosion (Bachtrog et al. 2014), which might reflect a requirement to maintain ancestral gene dosage and prevents dosage compensation systems from arising.

Chromosome-wide dosage compensation systems have been discovered in worms, flies, and mammals (• Fig. 4.2). In some species, distinct processes are involved in adjusting dosage difference between the sexes and dosage differences between sex chromosomes and the ancestral autosome pair. This is the case in mammals, which possess a single active $\mathrm{X}$ chromosome in both sexes. Transcriptional silencing of one of the two $\mathrm{X}$ chromosomes in female mammals by the process of $\mathrm{X}$ chromosome inactivation approximates gene expression of the single male $\mathrm{X}$ chromosome. In this case, a reduction in fitness due to emerging differences in gene dosage between the sexes appears to have driven the evolution of dosage compensation. The mechanism of $\mathrm{X}$ to autosome (X:A) compensation remains to be resolved in mammals. In contrast, in the fruit fly $D$. melanogaster the regulation of the sex chromosomes is accomplished in a manner that balances gene dosage between the sexes and restores the level of $\mathrm{X}$ chromosome expression in males relative to the ancestral autosome pair. This is facilitated through the transcriptional hyperactivation of genes on the single $\mathrm{X}$ chromosome in males, such that an expression level is achieved that corresponds to two $\mathrm{X}$ chromosomes in females. Thus, the evolution of the dosage compensation system in flies appears to be driven by the adjustment of X-linked expression, such that it maintains gene expression levels between the sexes as well as the expression level of the ancestral autosome pair. In the worm C. elegans, the two X chromosomes

1 Reduction of fitness caused by shifts in sex chromosomal to autosomal gene dosage is consistent with studies on human monosomies, which show that inheritance of a single autosome in an otherwise diploid genome has high fitness costs. Human monosomies are generally detrimental and only monosomy X, and monosomies of smaller parts of chromosomes 5 and 1 are viable. Trisomies are also detrimental and only trisomy 13, 18, and 21 are viable in humans leading to Patau, Edwards, and Down syndrome, respectively. 
- Fig. 4.2 Overview of dosage compensation in mammals, flies, and worms. X-linked gene expression differences between the sexes are compensated by different strategies. Mammals inactivate one of the two $\mathrm{X}$ chromosomes in female cells. In D. melanogaster the single male $\mathrm{X}$ chromosome is hyperactivated, and in C. elegans both $\mathrm{X}$ chromosomes are partially repressed in hermaphrodites female

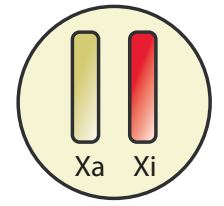

X chromosome inactivation

Drosophila

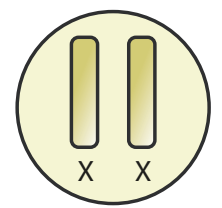

C. elegans
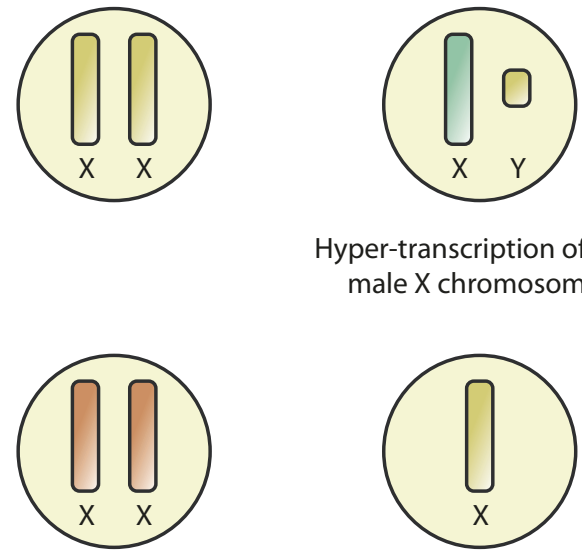

Hyper-transcription of the male X chromosome

Hypo-transcription of both $\mathrm{X}$ chromosmes in hermaphrodites

in hermaphrodites are partially repressed to approximate the gene expression level of the single $\mathrm{X}$ chromosome in males, which are XO. In C. elegans, it is again the dosage difference between the sexes that appears to drive the evolution of the dosage compensation mechanism. All studied dosage compensation systems to date have arisen within the context of an XY or XO sex chromosome system where the males carry the heteromorphic sex chromosome pair. Thus far, no chromosome-wide dosage compensation systems have been identified in ZW systems with heteromorphic female sex chromosomes. Consistent with the idea that X chromosomal dosage compensation in flies, worms, and mammals have evolved independently, these processes adopt molecularly distinct strategies and possess distinct genetic requirements.

\subsection{The Dosage Compensation Complex of the Fruit Fly Drosophila melanogaster}

The dosage compensation system of the fruit fly $D$. melanogaster has been extensively studied by genetic and biochemical approaches. D. melanogaster possess a XY sex 
chromosome system where a single $\mathrm{X}$ chromosome is present in male flies. ${ }^{2}$ The single male $\mathrm{X}$ chromosome is the target of a dosage compensation complex (DCC) which acts to enhance transcription and, thereby, approximates the expression from two X chromosomes in female flies. In this manner, not only the difference in X-linked gene expression between the sexes but also the ancestral X:A ratio is restored. Thus, the single male $\mathrm{X}$ chromosome emulates an ancestral pair of two $\mathrm{X}$ chromosomes. Conceptually, the single $\mathrm{X}$ chromosome needs to be identified to target transcriptional upregulation. In addition, dosage compensation needs to be regulated to prevent aberrant hyper-transcription of the two X chromosomes in females. In D. melanogaster, dosage compensation and sex determination are regulated by a common upstream pathway that senses the number of $\mathrm{X}$ chromosomes. If a single $\mathrm{X}$ chromosome is present, this pathway diverges to regulate male sex differentiation and independently induces to the formation of the DCC.

The components of the DCC have been identified in genetic screens for mutations that prevent the development of male flies. The Male specific lethal (Msl) factors Msl1, Msl2, and Msl2 as well as Male lethal (Mle) and Males absent on the first (Mof) are proteins that contribute to the formation of the DCC. The respective genes are genetically required for male but not female development. Notably, the Msl2 protein is only present in males but not in females. Msl2 is regulated by the sex determination cascade and is essential for DCC assembly. Its absence in females prevents erroneous dosage compensation of both $\mathrm{X}$ chromosomes. Thus, Msl2 presents a molecular link between sensing the presence of a single $\mathrm{X}$ chromosome and assembly of the DCC. Once the DCC is assembled in male embryos, it is targeted to multiple sites on the single $\mathrm{X}$ chromosome. These sites have been referred to as chromosome entry sites as they appear to confer independent affinity for the binding of the DCC. These sites have further been associated with the DNA-binding protein CLAMP. CLAMP appears to be required for association of the DCC with chromosome entry sites and has also other functions in addition to its role in dosage compensation. Because mutations affecting CLAMP are detrimental to both male and female development, it could not be identified as a component of the DCC in the original genetic screens.

The DCC spreads out from chromosome entry sites over the entire X chromosome. DCC spreading is dependent on non-coding RNAs and the RNA helicase Mle. The discovery that functional RNAs are essential components of the Drosophila dosage compensation system was made serendipitously. Two genes encoding $R N A$ on the $X 1$ (roXI) and rox2 were isolated as transcripts that are specifically expressed in males but not females. Their discovery was made considerably later than the identification of the protein components of the dosage compensation system. A likely reason why $r o X 1$ and $r o X 2$ were not discovered in genetic screens is that both RNAs are functionally redundant. Male development requires either roX1 or roX2. Only combined mutations of roX1 and roX2 lead to a failure of dosage compensation and male lethality. It is thought that Mle, which encodes an RNA helicase, mediates the association of roX1 and roX2 with the Msl complex. Association of Mle and roX

2 Drosophila males do possess a $\mathrm{Y}$ chromosome that contains a small number of genes but it does not specify male sex. Therefore, the $\mathrm{Y}$ chromosome is not relevant for sex determination in flies. 
- Fig. 4.3 Enrichment of H4K16ac on the X chromosome in D. melanogaster males. Immunostaining of a polytene chromosome spread with an antiserum specific for $\mathrm{H} 4 \mathrm{~K} 16 \mathrm{ac}$ identifies the $\mathrm{X}$ chromosome in males. Polytene chromosomes are obtained from salivary glands and contain many strands of DNA due to endoreduplication of the genome in this polyploid tissue. This makes them well-suited for microscopy studies. (From Bone et al. (1994))

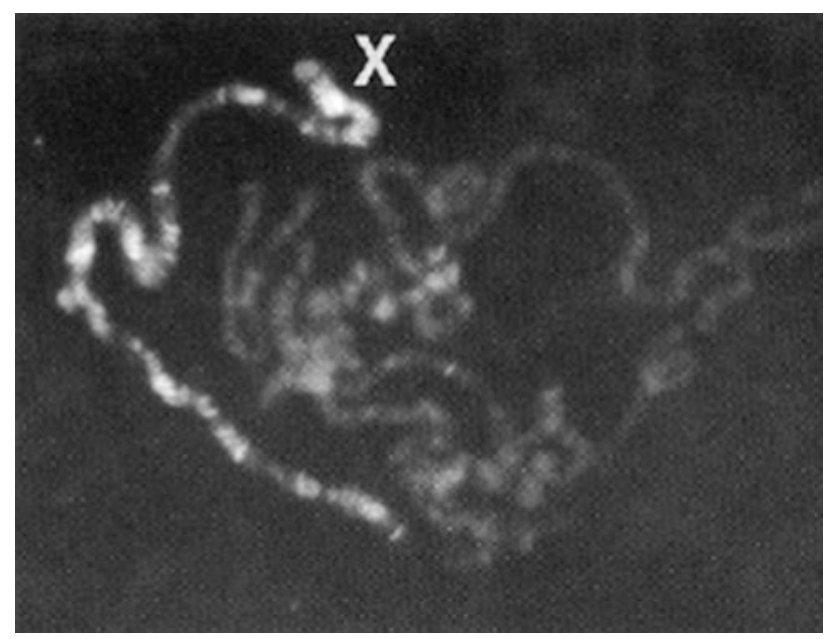

RNA thereby facilitates the spreading of dosage compensation from chromosome entry sites over the entire $\mathrm{X}$ chromosome.

Recruitment of the DCC to the X chromosome induces changes to chromatin and facilitates elevated transcription. An important component of the DCC that has been implicated in transcriptional regulation is the histone acetyltransferase Mof. Mof catalyzes the acetylation of histone $\mathrm{H} 4$ lysine 16 on the X chromosome in males (- Fig. 4.3). The activity of Mof thereby contributes to the transcriptional activation of the single male $\mathrm{X}$ chromosome, consistent with the idea that acetylation of lysines in histone tails has an activating role for gene expression and facilitates accessibility of the DNA (see book $>$ Chap. 1 of Wutz, and $>$ Chap. 2 of Paro). The relevance of the observation that lysine 16 is specifically targeted by Mof for dosage compensation remains to be further explored. Furthermore, it appears that histone acetylation on the $\mathrm{X}$ chromosome is not stable or heritable by itself but depends on the continued recruitment of the DCC (see $>$ Box 4.1). Depletion of Mof or Ms12 by RNA interference in cultured male $D$. melanogaster $\mathrm{S} 2$ cells was shown to cause a loss of histone H4 lysine 16 hyper-acetylation on the X chromosome (Zhang et al. 2010). Although, the mechanism of dosage compensation in flies has been well characterized, our understanding is far from complete and, certainly, additional components of the dosage compensation pathway will be identified in the future. In this context, it is interesting to note that the kinase JIL1 was found associated with the DCC but its role in dosage compensation remains unclear.

Box 4.1: A Conceptual Experiment: Testing the Heritability of Chromatin Marks on the D. melanogaster Male X Chromosome (• Box Fig. 4.1)

Depletion of MSL2 or MOF by RNA RNAi-mediated depletion ( $m s / 2$ and mof interference in S2 cells leads to loss of RNAi) and control (Mock) transfected H4K16ac on X-linked gene promoters. a S2 cells. b A schematic representation of A Western analysis shows MSL2 and H4K16ac enrichment over genes from MOF protein abundance following the $\mathrm{X}$ chromosome and the right arm of 
chromosome 3 as an autosomal control. H4K16ac enrichment was determined by ChIPseq (see book $>$ Chap. 1 of Wutz). The data are represented relative to DNA in the chromatin preparation to normalize for different sequence counts in different genomic regions. Each line represents a gene, the columns corre-

- Box Fig. 4.1 Depletion of msl2 and mof in $\mathrm{S} 2$ cells spond to S2 cells as indicated on top of panel a. Genes enriched (yellow) and depleted (blue) for H4K16ac are indicated.

S2 cells were derived from male fly embryos and possess a single $\mathrm{X}$ chromosome that is subject to dosage compensation. Conversely, the $\mathrm{X}$ chromosome is

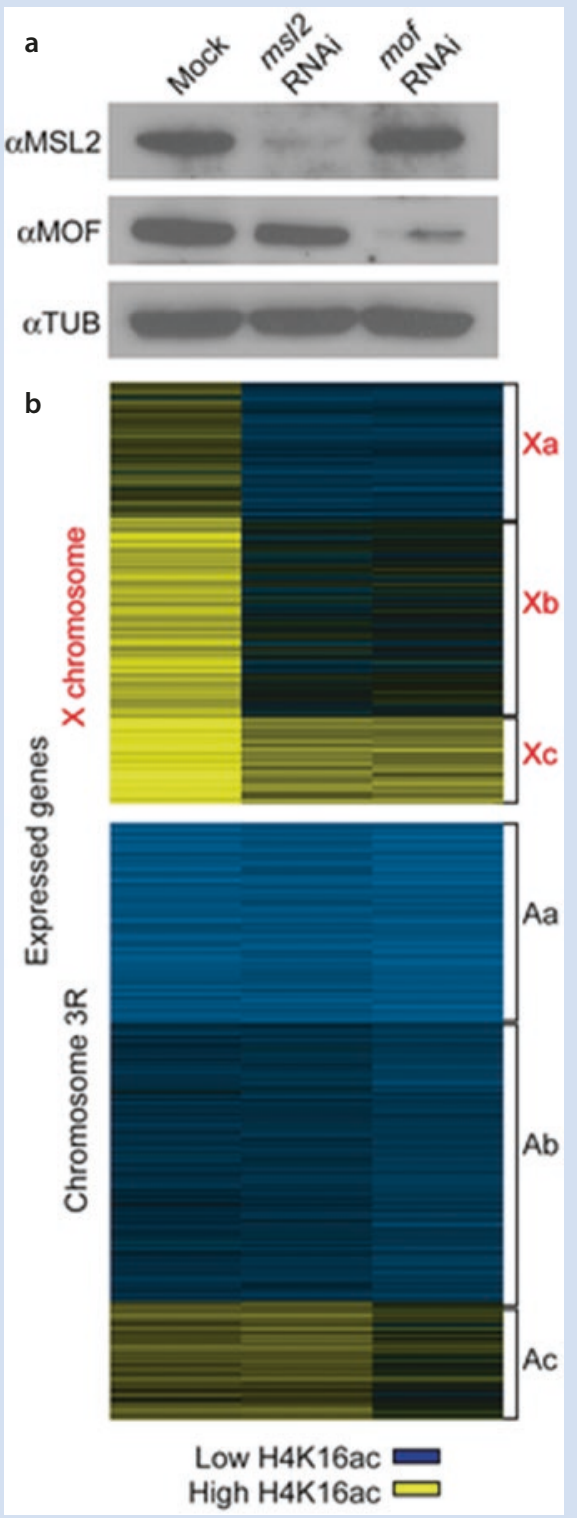


highly enriched for H4K16ac in control S2 cells. After depletion of MSL2 or MOF, this enrichment is lost and the $\mathrm{X}$ chromosome appears similar to chromo- some $3 \mathrm{R}$. This shows that the continuous activity of the DCC is required to maintain dosage compensation in $\mathrm{S} 2$ cells. (From Zhang et al. (2010))

The components of the D. melanogaster DCC have been investigated in distantly related insects to study the evolution of dosage compensation. An Msl complex is present in the distantly related insect Sciara (Ruiz et al. 2000) but localizes to all chromosomes in both sexes. The absence of sex-specific differences suggests that the Sciara Msl complex is not involved in dosage compensation and might have other functions. This points to a diversity of molecular mechanisms for dosage compensation among different insects. Interestingly, an independent chromosome-wide marking system has been observed on the fourth chromosome in D. melanogaster. The Painting of fourth (POF) protein associates with the fourth chromosome (• Fig. 4.4) where it enhances gene expression (Johansson et al. 2007). Initially, POF appeared to be unrelated to dosage compensation. However, a link between POF and an ancestral sex chromosome and dosage compensation system has recently been uncovered (Vicoso and Bachtrog 2013). The fourth chromosome of D. melanogaster is part of the sex chromosome in other fly species. POF is targeted to the male X chromosome in D. busckii and D. ananassae (Stenberg and Larsson 2011). In the latter, POF is recruited together with $\mathrm{H} 4 \mathrm{~K} 16 \mathrm{ac}$ and the $M s l$ complex. Overlap of chromatin marks of the $\mathrm{X}$ and fourth chromosome can be observed to varying degrees in different fly species. These observations could suggest a common origin of both pathways in an ancestral dosage compensation system and a potential separation of the $\mathrm{X}$ and forth chromosome in the lineage that led to the evolution of D. melanogaster. It is interesting to speculate that chromosome-wide modifications might be more common than would appear from the limited number of examples that are known today.

\subsection{Chromosome Inactivation in Mammals}

In mammals, dosage compensation of X-linked genes is achieved by repression of one of the two X chromosomes in female cells. X chromosome inactivation ensures that, in both sexes, a single $\mathrm{X}$ chromosome remains active. This mode of dosage compensation might appear less intuitive than the $D$. melanogaster system as the ances-

Fig. 4.4 Chromosome-wide marking of D. melanogaster $\mathrm{X}$ and fourth chromosomes. Immunostaining of POF (red) and $\mathrm{Msl} 3$ (green) of polytene chromosomes from $D$. melanogaster male salivary glands. (From Stenberg and Larsson (2011))

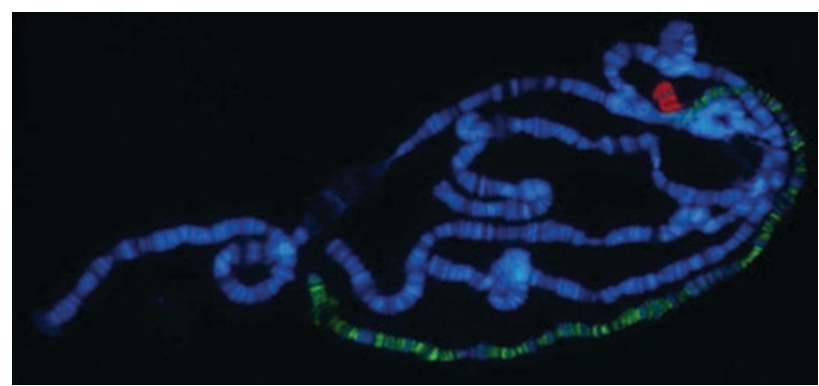


tral X:A gene expression ratio is changed from $2 \mathrm{X}: 2 \mathrm{~A}$ to $1 \mathrm{X}: 2 \mathrm{~A}$. The relative $\mathrm{X}: \mathrm{A}$ dosage shift associated with the evolution of the mammalian XY chromosome system could reduce fitness. In addition, mutations on the single $\mathrm{X}$ chromosome that is transcribed in both sexes are functionally hemizygous and cause phenotypes at a higher rate than recessive mutations on autosomes where complementation by the homolog is possible. Notably, random choice of which X chromosome is inactivated in placental mammals protects females from X-linked hemizygosity to some extent. Females comprise of a genetic mosaic of cells expressing either X-chromosome. Hence, in case of a heterozygous mutation, half of the cells will express the intact gene. However, it remains unknown to what extent the fitness costs arising from the evolution of the mammalian sex chromosome and dosage compensation systems have been absorbed. Although the evolution of X chromosome inactivation remains largely unclear, one potential scenario might be that it was derived from an extension of meiotic inactivation of unpaired chromosomes. Meiotic sex chromatin inactivation (MSCI) is a process that results in the transcriptional inactivation of unpaired chromosomes in meiosis during germ cell development. This process has been observed in a wide range of animal species. Inactivation is thought to allow unpaired chromosomes to be passed through meiosis. Reactivation of MSCI normally occurs after fertilization when embryo development starts. One might speculate that a perpetuation of MSCI of the paternal X chromosome might have evolved in mammals. As the paternal $\mathrm{X}$ chromosome is largely unpaired in male (XY) meiosis, silencing would be initiated by MSCI. In marsupial mammals, transcriptional inactivation of the paternally inherited $\mathrm{X}$ chromosome is observed, suggesting that imprinted $\mathrm{X}$ chromosome inactivation in marsupials might be based on mechanisms that extend silencing of MSCI. The process of random X chromosome inactivation in placental mammals poses a greater challenge to explain. Each female cell nucleus possesses two potentially genetically identical $\mathrm{X}$ chromosomes, whereby one is inactivated and the other remains active. Hence, both chromosomes assume divergent fates in the same nucleus. Therefore, a novel and complex mechanism had to be established at the split of placental and marsupial mammals.

\subsubsection{The Mammalian Dosage Compensation Mechanism}

The inactive X chromosome (Xi) was discovered serendipitously when a dark stained dot was observed in the nucleus of neurons from female cat brains (• Fig. 4.5). This dot was not present in male cat neurons (• Fig. 4.5). From this study, the Xi acquired its colloquial name "Barr body" in recognition of its discoverer (Barr and Bertram 1949). Subsequent studies revealed the identity of the Barr body as the Xi and the process of random $\mathrm{X}$ inactivation. A key insight into the mechanism was the identification of a transcript that is expressed only in female cells and encoded on the $\mathrm{X}$ chromosome. The $X$ inactive specific transcript (XIST) gene was initially identified in humans and shown to produce a functional RNA that associates with the Xi. XIST is located within a region of the $\mathrm{X}$ chromosome that contains additional regulators of dosage compensation which together make up the $\mathrm{X}$ inactivation center $(X I C)$. The $X I C$ encompasses elements that signal the presence of an $\mathrm{X}$ chromosome and regulate XIST expression if more than one is present. XIST expression precedes the transcriptional repression of genes on the $\mathrm{Xi}$. Therefore, XIST is an appealing solu- 

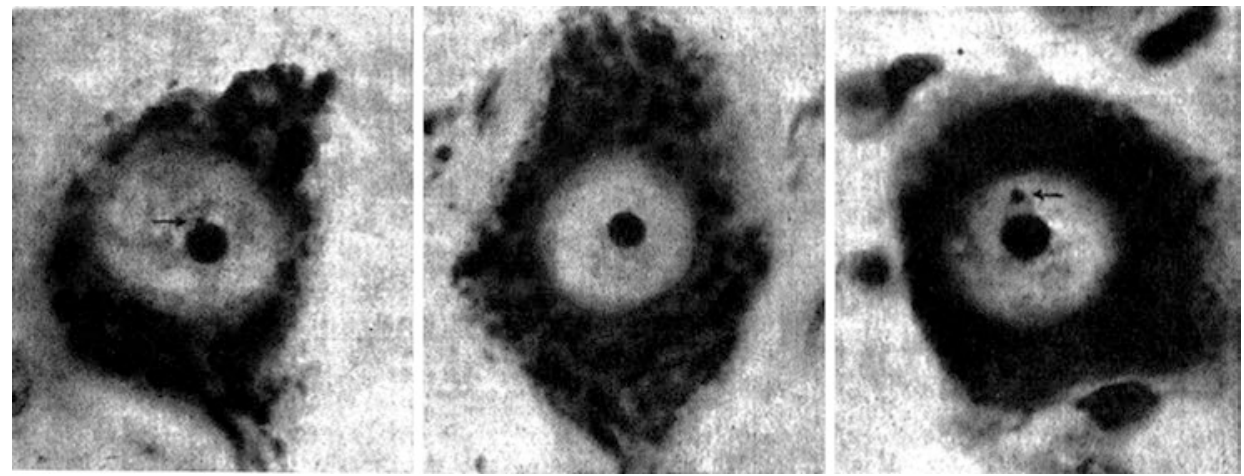

Fig. 4.5 The inactive $\mathrm{X}$ chromosome observed in neurons of female cats. Microscopy images of stained cat neurons show the cell darkly and the nucleus lightly stained. Within the nucleus, the large dark round spot is the nucleolus. Near the nucleolus, a smaller dark spot (arrow) can be observed in female neurons (left and right) but not in those of males (center). This is the inactive X chromosome which, in recognition of its discoverer, is also referred to as Barr body. (From Barr and Bertram (1949))

tion to restrict dosage compensation to one of the two $\mathrm{X}$ chromosomes in female cells. XIST specifically associates with the chromosome from which it is produced. The X chromosome that does not express XIST remains active. Since male cells contain only a single active $\mathrm{X}$ chromosome, they do not express XIST in general. Conceptually, the issue arises as to how XIST prevents its own repression on the $\mathrm{Xi}$, when the majority of gene promoters are inactivated. Actually, a number of genes are known that escape $\mathrm{X}$ inactivation and remain expressed from the $\mathrm{Xi}$. This observation points to the existence of specific expression control elements in the promoters of XIST and genes that escape $\mathrm{X}$ inactivation, but this aspect remains incompletely understood.

XIST localization to the $\mathrm{Xi}$ induces modifications of chromatin and gene repression. The formation of a fully inactivated $\mathrm{X}$ chromosome then occurs through sequential changes to Xi. Studies in mouse embryonic stem cells (ESCs), which recapitulate the process of $\mathrm{X}$ chromosome inactivation, have led to the view of a multistep process. Xist RNA recruits chromatin modifiers including Polycomb group (PcG) proteins and the mammalian homologue of Split ends (SPEN), an RNAbinding protein. Initially, repression and chromatin changes are dependent on XIST expression. Reactivation and complete reversion to an active $\mathrm{X}$ chromosome is observed when Xist is lost at this stage. This phase of $\mathrm{X}$ inactivation is referred to as the initiation phase, when Xist-dependent gene repression and chromatin changes are established that are reversible (• Fig. 4.6a). The initiation phase is distinguished from the maintenance of gene repression on $\mathrm{Xi}$ in differentiated somatic cells. During development, the $\mathrm{Xi}$ acquires a series of chromatin modifications as cells differentiate into mature cell types of the tissues. Chromatin changes on the $\mathrm{Xi}$ include the deposition of the histone $\mathrm{H} 2 \mathrm{~A}$ variant macroH2A, the loss of acetylation of histone $\mathrm{H} 4$, and the acquisition of DNA methylation at promoters of repressed genes. At this stage, Xist becomes dispensable for maintaining gene repression on the Xi. Therefore, the repressed state is perpetuated in a heritable manner without Xist. Stable maintenance of gene repression that is independent of XIST characterizes the maintenance phase of X chromosome inactivation ( $\bullet$ Fig. 4.6a). The fact that Xist, 


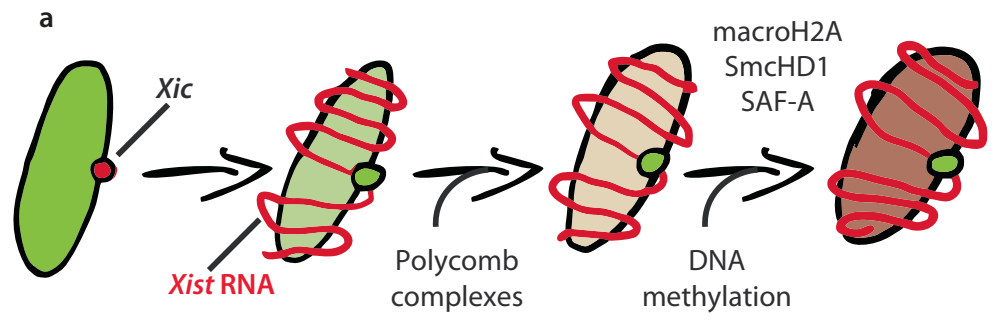

methylation

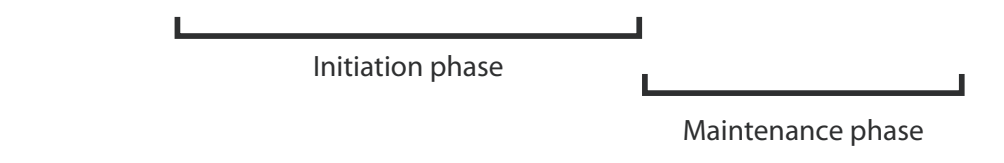

b
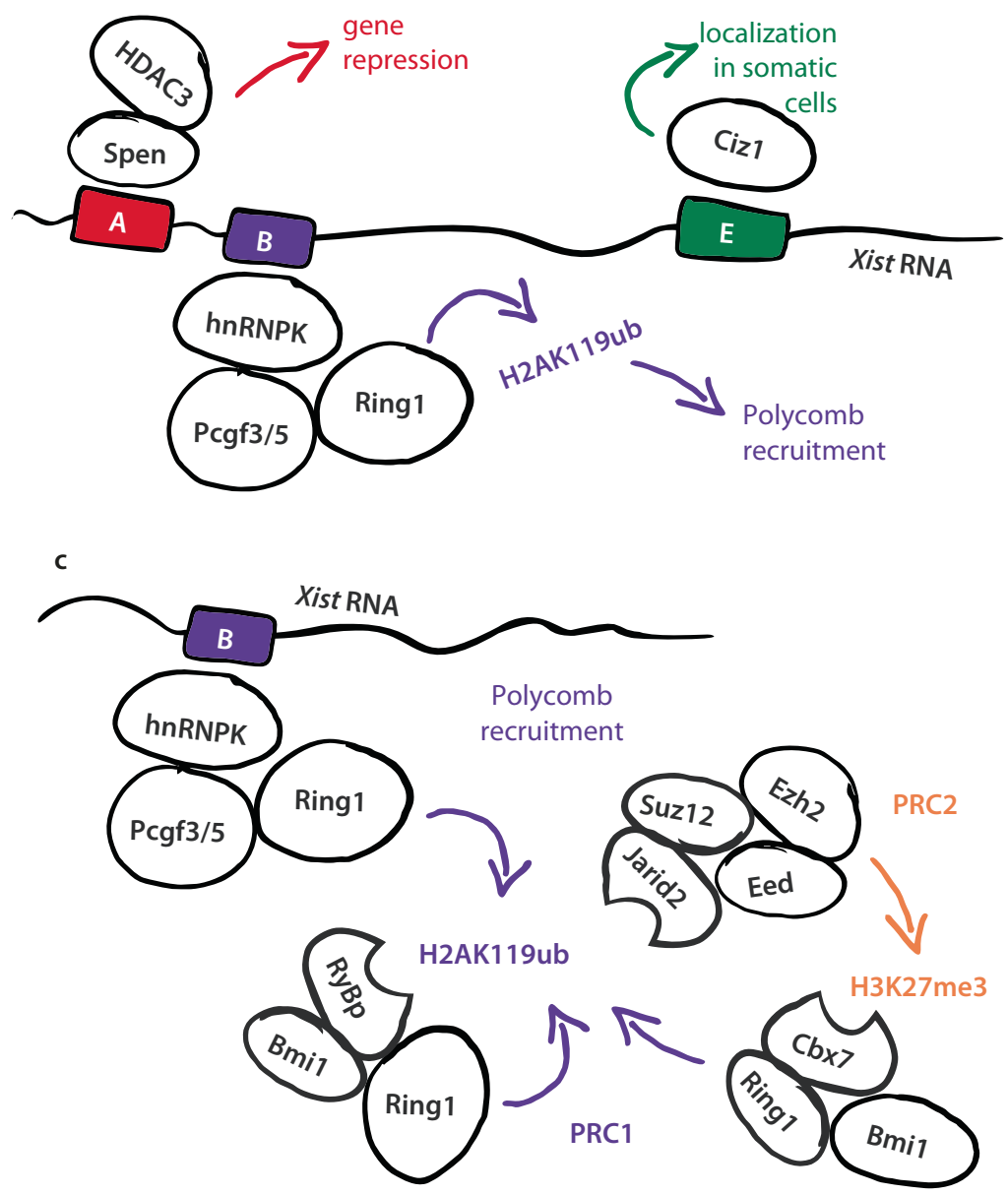

- Fig. 4.6 Chromosome-wide gene silencing during X chromosome inactivation. a Developmental changes of chromatin on the $\mathrm{Xi}$ are aligned with the initiation and maintenance phase of $\mathrm{X}$ chromosome inactivation. b Molecular interaction of Xist A-, B-, and E-repeats are indicated. $\mathbf{c}$ Recruitment of $\mathrm{PRC} 1$ and $\mathrm{PRC} 2$ in $\mathrm{X}$ chromosome inactivation is shown. These pathways lead to chromosome-wide modification of the $\mathrm{Xi}$ with $\mathrm{H} 3 \mathrm{~K} 27 \mathrm{me} 3$ and H2AK119ub 
which is the initial trigger of repression, is no longer needed for the maintenance phase, illustrates the characteristics of epigenetic memory (see book $>$ Chap. 3 of Paro).

$X I S T$ is a long RNA that lacks a reading frame encoding proteins. This observation, together with its localization over the $\mathrm{Xi}$, indicate that the functional product of the Xist gene is a transcript rather than a protein. A number of elements with repeated sequence motifs have been identified along Xist RNA. Deletion of one of these repeated elements at the $5^{\prime}$ end of Xist, which is referred to as Xist A-repeat, abolishes the function of Xist for gene repression (- Fig. 4.6b). In this case, many modifications of chromatin can be observed on the Xist-coated X chromosome independent of gene repression ( $\downarrow$ Box 4.2). This introduces a conceptual problem: how can genes remain active on a chromosome that carries chromatin modifications associated with heterochromatin? A potential explanation is the spatial separation of heterochromatin and genes. Genes appear to be positioned in the periphery of the $\mathrm{X}$ chromosome territory (CT), where they do not overlap with the domain of Xist RNA. Xist RNA establishes a domain enriched in heterochromatic modifications and forms a repressive compartment in the core of the CT (see book $>$ Chap. 1 of Wutz). Xist repeat-A is required for the repression of genes but not for the formation of the repressive compartment. Once genes are repressed, they associate with $\mathrm{Xi}$ heterochromatin. However, if repeat-A is deleted from Xist, repression of genes and association with the repressive compartment are not observed. These studies suggest that two types of chromatin are initially distinguished on the $\mathrm{Xi}$ and spatial organization of chromatin might play an important role during the initiation of $\mathrm{X}$ chromosome inactivation.

Box 4.2: A Conceptual Experiment: Separation of Gene Repression and Chromatin Modifications on the Mouse X Chromosome (• Box Fig. 4.2)

Expression of Xist RNA without the A-repeat leads to chromosome-wide chromatin changes but prevents the repression of X-linked genes. A heterologous expression system has been engineered in male ESCs to force expression of Xist without the A-repeat. a Staining with an antiserum specific for acetylated histone H4 (green) reveals a single hypoacetylated chromosome, which is identified as the $\mathrm{X}$ chromosome by DNA FISH using an $\mathrm{X}$ chromosome paint (red). b Histone H4 hypoacetylation is observed even after 9 days of differentiation, consistent with the observation that X-linked genes are expressed. A novel chromosome configuration that carries chromatin modifications associated with the $\mathrm{Xi}$ and active genes is displayed in this experiment. A magnified view of this Xiag (for $\mathrm{Xi}$ with active genes) is shown on the right. Notably, stripes of chromatin containing acetylated histone $\mathrm{H} 4$ can be discerned that would be missing from a silent $\mathrm{Xi}$. These stripes of active chromatin might represent clusters of active genes that are spatially separated from heterochromatin. (From Pullirsch et al. (2010)) 


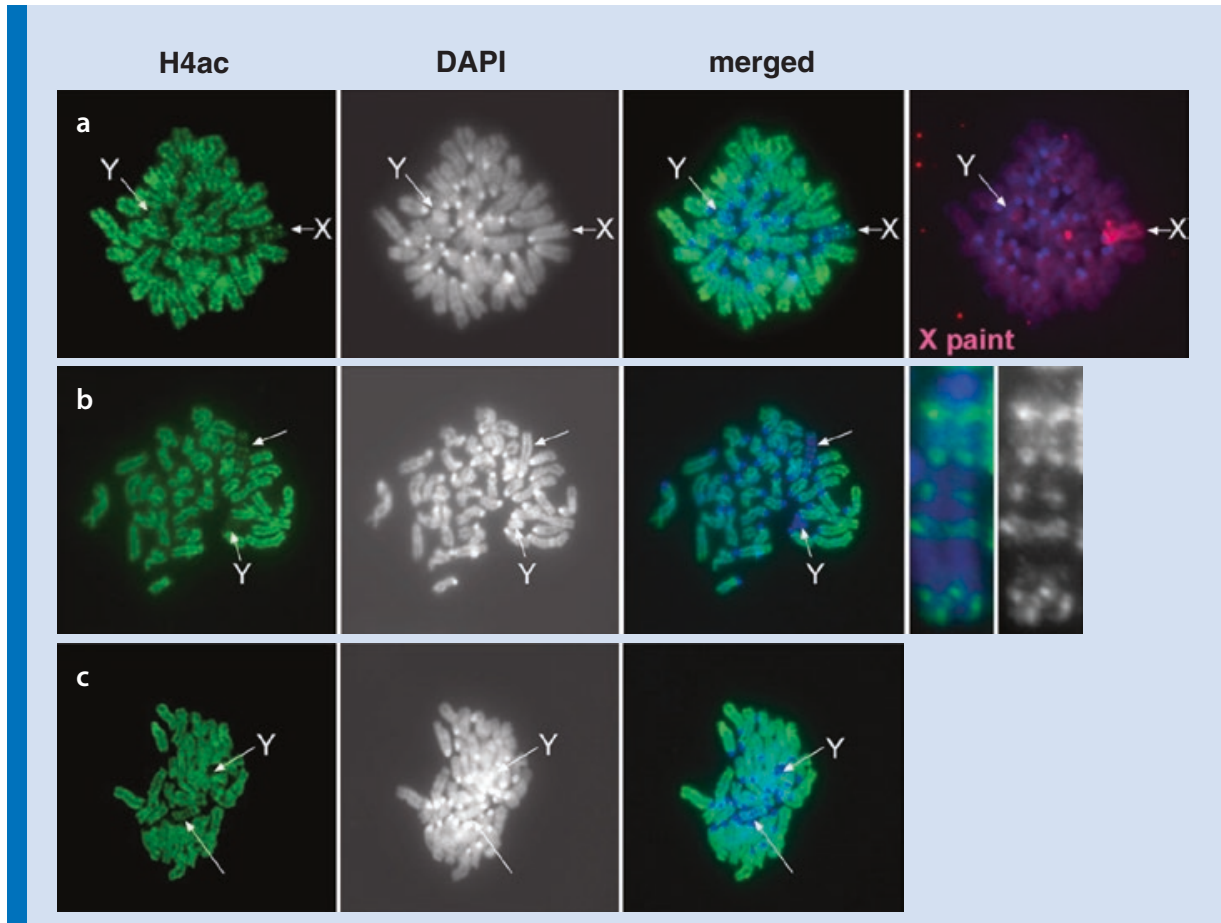

- Box Fig. 4.2 Chromosome-wide histone H4 hypoacetylation is triggered by Xist independent of gene silencing

The mechanism of gene repression by Xist has been investigated. The RNA-binding protein SPEN binds to the Xist A-repeat (- Fig. 4.6b) and is required for the function of Xist in gene repression. Mutations affecting SPEN phenocopy the deletion of Xist repeat-A in cultured mouse ESCs. SPEN associates with NCoR/SMRT corepressor complexes, which contain the histone deacetylase HDAC3. The latter suggests that histone deacetylation contributes to the gene silencing pathway of Xist. A conceptually important question is how Xist function in repressing genes can be localized to the $\mathrm{X}$ chromosome from which it is expressed. The RNA binding protein hnRNPU is required for localizing Xist RNA to Xi in specific cellular contexts. Notably, hnRNPU is a protein that associates with the nuclear scaffold and is also referred to as Scaffold attachment factor A (SAF-A). Interactions with nonchromatin nuclear organization seems to contribute to the specific localization of Xist to the Xi. Recently, the cyclin binding protein CIZ1 has also been shown to be required for Xist localization in differentiated somatic cells but not in embryonic cells. CIZ1 is enriched at the $\mathrm{Xi}$, apparently mediated by the Xist repeat-E (- Fig. 4.6b).

During the initiation phase of $\mathrm{X}$ chromosome inactivation, $\mathrm{PcG}$ proteins (see book Chap. 3 of Paro) are recruited to the Xi. This Xist RNA-dependent recruitment mechanism has been analyzed in detail. The RNA-binding protein hnRNPK is required for $\mathrm{PcG}$ recruitment to the $\mathrm{Xi}$. Xist repeat-B and repeat-C have been shown 
to represent binding sites for hnRNPK. Initially, a complex containing the PcG proteins PCGF3 or PCGF5 and the catalytic subunit of Polycomb Repressive Complex 1 (PRC1), RING1B (• Fig. 4.6b), is recruited by hnRNPK to Xist. This atypical PRC1 complex mediates ubiquitinylation of histone H2A lysine 119 (H2AK119ub). This modification has two functions. Firstly, it can recruit additional PRC1 complexes that contain RyBb which has affinity for H2AK119ub. Secondly, Jarid2 binds H2AK119ub and activates PRC2. PRC2 contains the histone methyltransferase EZH2 and catalyzes tri-methylation of histone H3 lysine 27 (H3K27me3) on the Xi. $\mathrm{H} 3 \mathrm{~K} 27 \mathrm{me} 3 \mathrm{can}$, in turn, enhance the recruitment of PRC2 through the EED protein, which has affinity for $\mathrm{H} 3 \mathrm{~K} 27 \mathrm{me} 3$. In addition, recruitment of canonical PRC1 complexes is mediated through the CBX7 subunit, which contains a chromodomain with affinity for $\mathrm{H} 3 \mathrm{~K} 27 \mathrm{me}$. Multiple interactions between $\mathrm{PcG}$ proteins and histone modifications generate a feed-forward loop that results in a strong enrichment of PcG proteins over Xi (• Fig. 4.6b). Spreading of PcG complexes leads to a high amount of histone modifying activity and, therefore, nearly all histone $\mathrm{H} 3$ on the $\mathrm{Xi}$ carry the H3K27me3 modification. Multiple interactions between PcG complexes endow this chromatin modification system with a substantial robustness. Random $\mathrm{X}$ chromosome inactivation appears not materially affected when individual PcG proteins are mutated. A mutation in the EED gene diminishes PRC2 activity and blocks the establishment of $\mathrm{H} 3 \mathrm{~K} 27 \mathrm{me} 3$ on $\mathrm{Xi}$ but not the recruitment of PRC1 complexes, establishment of H2AK119ub, and gene repression. Furthermore, additional requirements for the recruitment of PcG proteins to $\mathrm{Xi}$ appear to exist. For example, a depletion of SPEN has been shown to reduce the amount of PcG complexes that are recruited by Xist. However, it is not clear whether SPEN directly interacts with PcG complexes or indirectly causes an effect on PcG recruitment by influencing gene repression or Xist RNA modification. It is noteworthy that a deletion of Xist repeatA appears to have less effect on PcG recruitment by Xist than the mutation of SPEN. Future studies will be required to explain these observations.

Additional factors that contribute to the initiation of $\mathrm{X}$ chromosome inactivation include lamin B receptor (LBR) and a complex that mediates methylation of adenines on RNA. It is likely that several of these molecular pathways act in parallel and, through their combination, bring about the remarkable localization and repressive function of Xist RNA. Conceptually, it is tempting to speculate that Xist RNA might contain multiple binding sites for different complexes, repeat-A, $-\mathrm{B}$ and $-\mathrm{C}$, and $-\mathrm{E}$ are examples in support of this view (• Fig. 4.6b). Thus, $\mathrm{X}$ inactivation might instantiate novel combinatorial functions of molecular components that have other roles in distinct biological processes.

The maintenance of $\mathrm{X}$ chromosome inactivation involves other factors than its initiation. The atypical structural maintenance of chromosomes (SMC) protein SMC hinge domain 1 (SmcHD1) has been identified in a genetic screen in mice. A mutation in SmcHDl causes female-specific lethality, suggesting a role in dosage compensation (Blewitt et al. 2008). Subsequent studies could show that SmcHDI is required for the maintenance of gene repression and promoter DNA methylation in somatic cells. In contrast, the initiation of $\mathrm{X}$ chromosome inactivation is largely unaffected by a mutation in SmcHD1. SmcHD1 is conceptually important as it is a rare example of a gene whose mutation causes sex-specific lethality in mammals (another one being Xist). Many components of the dosage compensation system 
have additional functions that are also required in males. For example, the $h n R N P K$ mutation is embryonic lethal in mice of both sexes (Gallardo et al. 2015).

Although most of the genes on the $\mathrm{Xi}$ are transcriptionally silenced, certain genes remain active on the $\mathrm{Xi}$. Some of these genes are located in regions of the $\mathrm{X}$ chromosome that are homologous with the $\mathrm{Y}$ chromosome and exchange genetic information through recombination during meiosis in male germ cells through $\mathrm{X}-\mathrm{Y}$ pairing. Genes within these pseudoautosomal regions (PARs) of the $X$ chromosome are present in two copies in both sexes, similar to autosomal genes. Therefore, these genes do not require dosage compensation and remain transcriptionally active on the Xi. In addition, several genes that do not possess homologues on the $\mathrm{Y}$ chromosome are expressed from the $\mathrm{Xi}$. These genes are referred to as escape genes and the phenomenon is called "escape from X inactivation". Escape genes are important for understanding phenotypes that arise from numerical aberrations of sex chromosomes including the human Klinefelter (XXY) and Turner (XO) syndromes. The level of expression of escape genes from the $\mathrm{Xi}$ often does not reach that of the $\mathrm{X}$ chromosome that remains active (Xa). Furthermore, for some genes, tissue-specific escape or polymorphic escape among individuals has been observed (Carrel and Willard 1999, 2005). The molecular features that determine whether a gene is repressed or remains active on the Xi remain to be explored further. Notably, it appears that a higher frequency of escape genes can be observed in regions of the $\mathrm{X}$ chromosome that were recently acquired from autosomal fusions. This correlation could indicate that chromosomal features change in evolution to accommodate dosage compensation.

\subsubsection{Regulation of $\mathrm{XCl}$ in Different Mammals}

The Xist gene originated before the radiation of placental mammals ${ }^{3}$ (• Fig. 4.1). However, mammalian sex chromosomes and dosage compensation systems have continued to evolve after the dosage compensation system was established. Even today, evolution continues to introduce changes into sex chromosome and dosage compensation systems among placental mammals, which can be illustrated by striking examples. Dominant mutations that specify female fate have arisen on the $\mathrm{X}$ chromosomes of lemmings and African pygmy mice (Veyrunes et al. 2010). As a consequence, females that possess one $\mathrm{X}$ chromosome carrying a dominant modifier and a $\mathrm{Y}$ chromosome $\left(\mathrm{X}^{*} \mathrm{Y}\right.$ females) can be observed along with $\mathrm{XX}$ and $\mathrm{XX}^{*}$ females, and $\mathrm{XY}$ males. Possibly, the increased ratio of females over males was favored in these species. In contrast, novel autosomal male sex determining factors can be observed in specific vole species (Mulugeta et al. 2016). A novel autosomal sex determination mechanism has led to the loss of the second sex chromosome in voles of the species Ellobius lutescens. A single X chromosome is present in both males and females and likely $\mathrm{X}$ chromosome inactivation is no longer initiated. E. talpinus and E. tancrei possess two $\mathrm{X}$ chromosomes in both sexes and have lost their $\mathrm{Y}$ chromosomes entirely. It is likely that $\mathrm{X}$ chromosome inactivation occurs in both sexes of

3 Evolutionary radiation of placental mammals followed the extinction of the dinosaurs 65 million years ago. In the late Cretaceous, placental mammals were mostly represented by small shrew-like animals from which such diverse species as mice, bats, dolfins, cows, dogs, and humans evolved. 
these species. These examples illustrate dramatic changes in sex determination strategies between closely related species. They are accompanied by a gain or loss of heteromorphic sex chromosomes and dosage compensation processes. The presence of an inactive $\mathrm{X}$ chromosome in E. talpinus males and females bears some reminiscence of the modified fourth chromosome in D. melanogaster as an evolutionary relict of a past sex chromosome system.

In placental mammals, chromosome-wide silencing is a consequence of Xist expression. The regulation of Xist is, therefore, important for correct dosage compensation whereby, in males, a single $X$ chromosome remains active and, in females, one of the two $\mathrm{X}$ chromosomes is inactivated. Conceptually, it has been proposed that blocking factors would prevent Xist activation in male cells. In female cells, $\mathrm{X}$-linked activation factors would be produced from two X chromosomes in sufficient amounts to counteract the blocking factors and lead to Xist expression. Blocking factors are expected to be encoded on autosomes and to set a threshold in male and female cells for X-linked activation factors to induce Xist expression. The identity of these blocking factors and X-linked activators remains to be fully understood but several genes with predicted properties have been identified (• Fig. 4.7a). These genes appear to be species-specific, likely reflecting the evolutionary divergence of placental mammals.

In mice, the X-linked gene Rnf12/RLim can trigger Xist expression in male ESCs when it is expressed from transgenes. This observation suggests that Rnf12/RLim is an X-linked activator of $\mathrm{X}$ chromosome inactivation. The function of Rnf12/RLim in female mouse embryos appears to be restricted to the initiation of imprinted $\mathrm{X}$ inactivation in early embryos (• Fig. 4.7b). Therefore, it has been proposed that other activators might act in the regulation of random $\mathrm{X}$ chromosome inactivation in mice.

Also, the mechanism of blocking factor function has been studied. A potential gene that likely acts downstream of blocking factors and negatively regulates Xist expression is the non-coding RNA gene Tsix, whose name is derived from the reverse spelling of Xist because it is transcribed in antisense orientation to Xist (• Fig. 4.7a). Tsix represses the promoter of Xist on the same chromosome from which it is expressed and, therefore, can be considered a cis-acting repressor. Tsix is the target of autosomal factors that enhance Tsix transcription and can be considered as blocking factors (- Fig. 4.7a). Among these factors are several transcription factors that are expressed in early embryos and repress Xist. These factors are not exclusively functioning in dosage compensation but have also other roles in embryo development.

For understanding the regulation by blocking factors, it is helpful to examine the function of the Tsix and $X A C T$ genes in mice and humans, respectively. Both genes are $\mathrm{X}$-linked and produce non-coding transcripts. They are cis-acting repressors of $X i s t$ and their expression is associated with Xa. Yet, Tsix and XACT are not conserved between mouse and human and likely act by different molecular mechanisms. $X A C T$ encodes a non-coding transcript that associates with the chromosome territory of the $\mathrm{Xa}$ and appears to counteract XIST localization and gene repression. In humans, both $\mathrm{X}$ chromosomes in female cells initially express XIST but X-linked gene silencing appears to be incomplete. The reduced gene expression caused by this early wave of XIST expression is referred to as "dampening" instead of silencing of $\mathrm{X}$-linked gene expression and is exclusively observed in humans. It is conceivable that antagonistic interactions between $X A C T$ and XIST contribute to dampening. As 
a
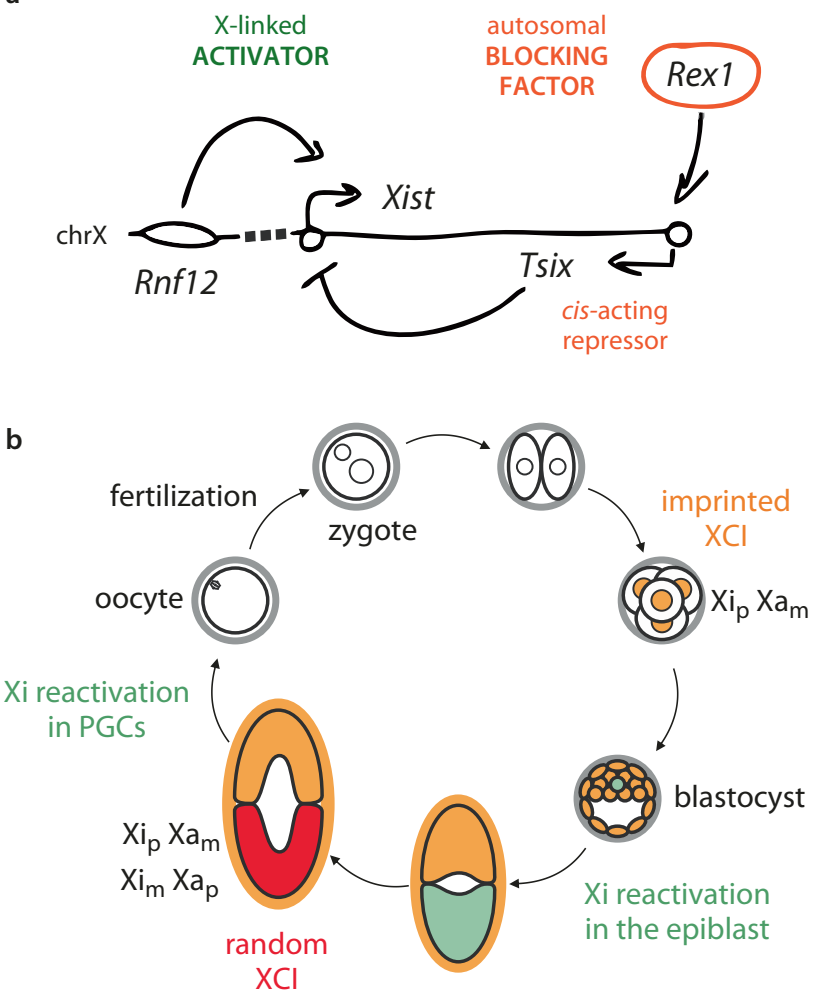

- Fig. 4.7 Regulation of X chromosome inactivation in mice. a Xist expression is stimulated by $\mathrm{X}$-linked activators, including Rnf12. Blocking factors enhance expression of Tsix that is a cis-acting repressor of Xist. In males, X-linked activators are not abundant enough to overcome the repressive effect of blocking factors and $\mathrm{X}$ inactivation is not initiated. b Imprinted (orange) and random (red) $\mathrm{X}$ chromosome inactivation in mouse embryos. Initially, the paternally inherited X chromosome in inactivated in 4-cell embryos. The $\mathrm{Xi}$ is maintained in the extraembryonic membranes and placenta but reactivated in the epiblast. After implantation, random inactivation of the maternal or paternal $\mathrm{X}$ chromosome is initialed in the cells of the developing embryo. Once the $\mathrm{Xi}$ is chosen, all descendants of the cell maintain this choice, giving rise to a genetic mosaic of patches of cells

embryo development progresses, one $\mathrm{X}$ chromosome maintains XIST expression and initiates gene silencing whereas the second $\mathrm{X}$ chromosome remains active and is covered by $X A C T$. $X A C T$ expression ceases as cell differentiation progresses further, suggesting a role for $X A C T$ specifically in regulating the initiation phase of $\mathrm{X}$ inactivation. The observation that independent cis-acting repressors of Xist have evolved in mice and humans suggests a model where blocking factors activate these X-linked RNAs, thereby counteracting the initiation of $\mathrm{X}$ inactivation. If this view were correct, blocking factor abundance or activity would be integrated at the promoters of $T$ six and $X A C T$.

In marsupials, inactivation of the paternally inherited $X$ chromosome is observed. Imprinted $\mathrm{X}$ inactivation is distinct from random $\mathrm{X}$ inactivation that is exclusive to placental mammals (• Fig. 4.1). In the American opossum Monodelphis domestica, $R s x$ appears to possess a similar function as Xist in mice (Grant et al. 2012). Rsx and 
Xist encode non-coding RNAs that associate with the Xi chromosome territory. Xist has evolved from the $\operatorname{Ln} x 3$ gene, which is a protein-coding gene in extant vertebrates (Duret et al. 2006). In contrast, Rsx seems to have arisen from a gene in proximity to the Hprt gene that is distinct from Lnx3, which remains protein-coding in marsupials. Therefore, Xist and Rsx are likely products of convergent evolution as the dosage compensation mechanisms of marsupials and placental mammals share a number of similarities. Both mechanisms include non-coding RNAs and involve the acquisition of H3K27me3 over repressed X-linked genes (Wang et al. 2014). It could be speculated that the molecular basis for this dosage compensation mechanism existed before the split of marsupial and placental mammals and had emerged likely soon after the split from monotremes. Divergence might have subsequently led to the origin of Xist and $R s x$ in independent phylogenetic lineages (• Fig. 4.1). In contrast, dosage compensation in platypus appears partial and gene-specific, similar to dosage compensation in birds.

\subsection{Chromosome Dosage Compensation in Caenorhabditis elegans}

C. elegans reproduces through hermaphrodites that possess two $\mathrm{X}$ chromosomes and produce sperm and eggs. Environmental triggers can induce the production of males with a single $\mathrm{X}$ chromosome but no $\mathrm{Y}$ chromosome. Dosage compensation in the worm equalizes $\mathrm{X}$-linked gene expression to the single $\mathrm{X}$ chromosome in males. For this, genes on both $\mathrm{X}$ chromosomes are repressed in hermaphrodites. C. elegans possesses a dosage compensation complex (DCC) that contains structural maintenance of chromosomes (SMC) proteins and resembles a condensin-like complex (see book - Chap. 1 of Wutz). Therefore, the composition of the fly and worm DCCs are different and, in the worm, the DCC is recruited to both X chromosomes of hermaphrodites. DCC recruitment leads to an impairment of transcription to reduce the gene expression level by half to match that of the single male $\mathrm{X}$.

Components of the $C$. elegans DCC have been identified by screening for sexspecific lethal mutations. Whereas defects in dosage compensation cause male lethality in D. melanogaster, they lead to lethality of hermaphrodites in C. elegans. The SMC protein MIX-1, together with several subunits that are shared with condensin, make up the DCC (• Fig. 4.8). The SMC family of proteins has functions in organizing chromatin during cell division, coordination of gene expression, and DNA repair. In the case of the C. elegans DCC, SMC proteins mediate a partial repression of transcription on both $\mathrm{X}$ chromosomes. As a consequence of DCC localization, $\mathrm{H} 4 \mathrm{~K} 20 \mathrm{me} 1$ is enriched on the two $\mathrm{X}$ chromosomes.

In C. elegans, the number of $\mathrm{X}$ chromosomes determines sex and initiates dosage compensation (- Fig. 4.8). The DCC is engaged by SDC-2, which also functions to suppresses male development. SDC-2 localizes to both $\mathrm{X}$ chromosomes together with the DCC. The dosage compensated X chromosomes contain low levels of H4K16ac. Recent studies provided evidence that deacetylation of H4K16ac can already be observed early in worm development before the DCC is recruited. This early deacetylation seems to require a PRC2-like complex that contains MES2, a homologue of E(z), MES3, and MES6. This suggests the presence of an initiation 


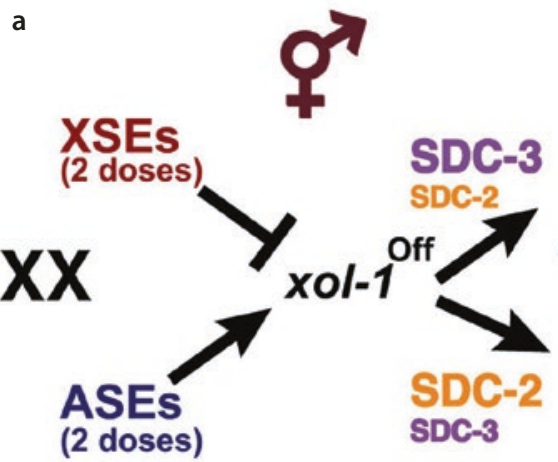

b

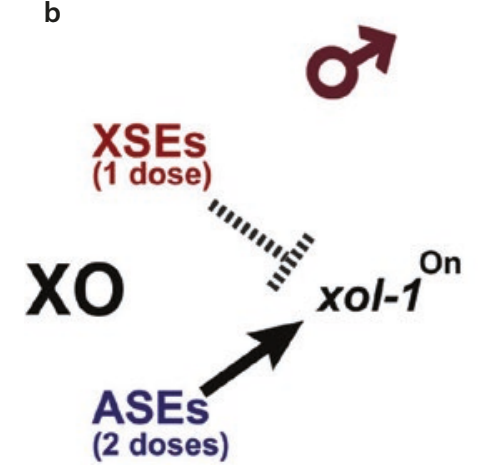

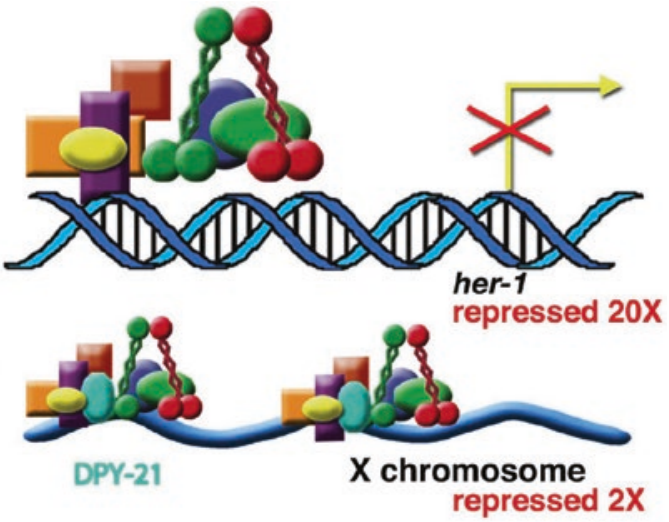

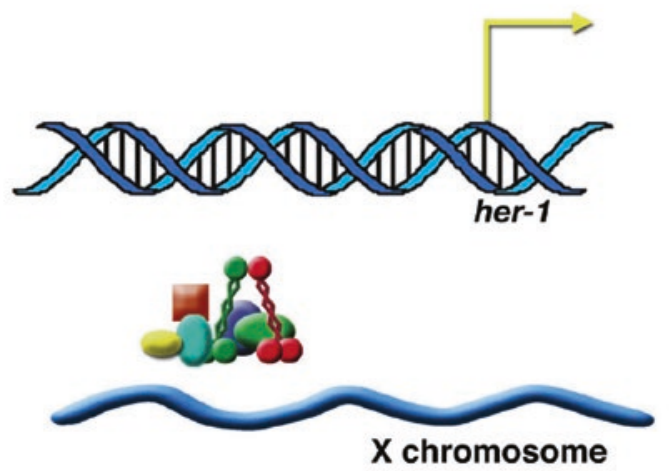

- Fig. 4.8 Genetic control of sex determination and dosage compensation in C. elegans. a xol-1 is the master sex-determination gene that controls both sex determination and dosage compensation. It is the direct molecular target of the X-chromosome counting mechanism that determines sex. In XX hermaphrodites, two doses of the X-signal elements (XSEs) repress xol-1 by overcoming the xol-1 activation achieved by autosomal signal elements (ASEs). xol-1 repression occurs at the level of transcription and pre-mRNA splicing. When xol-1 is repressed, the XX-specific gene $S D C-2$ is active and stabilizes SDC-3. SDC-2 acts with SDC-3 to target dosage compensation proteins to the $\mathrm{X}$ chromosome, thereby repressing gene expression by half. SDC-2 plays the lead role in recognizing X-specific sequences. It is the only dosage compensation protein expressed solely in XX animals. SDC-2 also activates the hermaphrodite program of sexual development by repressing the male-specific sex-determination gene her1. SDC-2 acts with SDC-3 to recruit dosage compensation proteins to her-1. In this case, SDC-3 plays the lead role in recognizing her-1 DNA targets. The her-1 and X complexes differ by one component: DPY-21 is present on the X but not at her-1. b In XO males, the single dose of XSEs is insufficient to overcome the activating influence of ASEs. xol-1 is active and promotes male fate by repressing the activities of $s d c$ genes. her-1 is transcribed and the single $\mathrm{X}$ is not repressed. In xol-1 XO mutants, the dosage compensation complex localizes to the single $\mathrm{X}$ chromosome, killing $\mathrm{XO}$ animals by reducing X-linked gene expression. (Copyright: (C) 2005 Barbara J. Meyer. From Meyer, B. J. X-Chromosome dosage compensation (June 25, 2005), WormBook, ed. The C. elegans Research Community, WormBook, doi/ $\triangleright$ https://doi.org/10.1895/wormbook.1.8.1, \ http://www.wormbook.org., which permits unrestricted use, distribution, and reproduction in any medium, provided the original author and source are credited) 
and maintenance phase in C. elegans dosage compensation. The DCC is required to maintain partial repression and reduced H4K16ac on the $\mathrm{X}$ chromosomes. These observations suggest some similarities to dosage compensation in mammals, where the Xi shares features including H4K20mel enrichment, histone H4 hypoacetylation, as well as PRC2 recruitment. Notably, SmcHD1 is involved in maintenance of the Xi and shares the hinge domain of SMC proteins. Although hypo-transcription of $\mathrm{X}$ chromosomes in C. elegans hermaphrodites is superficially similar to dampening of $\mathrm{X}$-linked gene expression in early human embryos, any mechanistic similarity remains to be demonstrated. Thus far, no RNAs have been implicated in dosage compensation in $C$. elegans, where both $\mathrm{X}$ chromosomes in hermaphrodites appear to be recognized through DNA motifs. Similar to mammals, dosage compensation is initiated in $C$. elegans in a developmentally regulated manner. Thus, repression of both $\mathrm{X}$ chromosomes is observed in the germline and involves a PRC2-like complex and $\mathrm{H} 3 \mathrm{~K} 27 \mathrm{me}$. This initial repressive mechanism could persist to some extent into the embryo and mark the early phase of X chromosome modification. Future studies will show to what extent mechanisms overlap for X-linked gene repression in C. elegans and mammals.

\section{Take-Home Message}

- The dosage compensation systems of flies, worms, and mammals engage different mechanisms to achieve chromosome-wide adjustments of gene expression between the sexes.

- In the fruit fly D. melanogaster, a dosage compensation complex mediates the upregulation of $\mathrm{X}$-linked genes in males to adjust gene dosage to the level of two $\mathrm{X}$ chromosomes as is observed in females.

- In mammals, the process of X chromosome inactivation silences one of the two X chromosomes in female cells, thereby equalizing the gene dose to the single $\mathrm{X}$ chromosome of males.

- In the nematode C. elegans, the two X chromosomes in hermaphrodites are partially repressed and adjust the gene dose to a level similar to males that possess a single $\mathrm{X}$ chromosome.

- Components of dosage compensation systems have functions in animal development in addition to dosage compensation and can, therefore, be considered model systems for understanding gene regulation.

- The fly and mammalian dosage compensation systems include long non-coding RNAs (Xist, Rsx, XACT, roX1, and roX2) as part of the mechanism.

- Chromosome-wide dosage compensation mechanisms have, thus far, been discovered in XY systems in a few animal species, although sex chromosome systems are widely distributed among animals and in dioecious plants (Vyskot and Hobza 2015). It is possible that novel molecular reagents facilitate further discoveries. Considering past discoveries made in dosage compensation systems, future research in this area promises to extend our understanding of the molecular mechanisms and the evolution of pathways for gene regulation. 


\section{References}

Bachtrog D, Mank JE, Peichel CL, Kirkpatrick M, Otto SP, Ashman TL, Hahn MW, Kitano J, Mayrose I, Ming R, Perrin N, Ross L, Valenzuela N, Vamosi JC, Tree of Sex C (2014) Sex determination: why so many ways of doing it? PLoS Biol 12(7):e1001899. https://doi.org/10.1371/journal.pbio.1001899

Barr ML, Bertram EG (1949) A morphological distinction between neurones of the male and female, and the behaviour of the nucleolar satellite during accelerated nucleoprotein synthesis. Nature 163(4148):676

Bellott DW, Skaletsky H, Pyntikova T, Mardis ER, Graves T, Kremitzki C, Brown LG, Rozen S, Warren WC, Wilson RK, Page DC (2010) Convergent evolution of chicken Z and human X chromosomes by expansion and gene acquisition. Nature 466(7306):612-616. https://doi.org/10.1038/nature09172

Blewitt ME, Gendrel AV, Pang Z, Sparrow DB, Whitelaw N, Craig JM, Apedaile A, Hilton DJ, Dunwoodie SL, Brockdorff N, Kay GF, Whitelaw E (2008) SmcHD1, containing a structuralmaintenance-of-chromosomes hinge domain, has a critical role in $\mathrm{X}$ inactivation. Nat Genet 40(5):663-669. https://doi.org/10.1038/ng.142

Bone JR, Lavender J, Richman R, Palmer MJ, Turner BM, Kuroda MI (1994) Acetylated histone H4 on the male $\mathrm{X}$ chromosome is associated with dosage compensation in Drosophila. Genes Dev 8(1):96104

Carrel L, Willard HF (1999) Heterogeneous gene expression from the inactive X chromosome: an $\mathrm{X}$-linked gene that escapes $\mathrm{X}$ inactivation in some human cell lines but is inactivated in others. Proc Natl Acad Sci U S A 96(13):7364-7369

Carrel L, Willard HF (2005) X-inactivation profile reveals extensive variability in X-linked gene expression in females. Nature 434(7031):400-404. https://doi.org/10.1038/nature03479

Duret L, Chureau C, Samain S, Weissenbach J, Avner P (2006) The Xist RNA gene evolved in eutherians by pseudogenization of a protein-coding gene. Science 312(5780):1653-1655. https://doi. org $/ 10.1126 /$ science. 1126316

Gallardo M, Lee HJ, Zhang X, Bueso-Ramos C, Pageon LR, McArthur M, Multani A, Nazha A, Manshouri T, Parker-Thornburg J, Rapado I, Quintas-Cardama A, Kornblau SM, Martinez-Lopez J, Post SM (2015) hnRNP K is a haploinsufficient tumor suppressor that regulates proliferation and differentiation programs in hematologic malignancies. Cancer Cell 28(4):486-499. https://doi. org/10.1016/j.ccell.2015.09.001

Grant J, Mahadevaiah SK, Khil P, Sangrithi MN, Royo H, Duckworth J, McCarrey JR, VandeBerg JL, Renfree MB, Taylor W, Elgar G, Camerini-Otero RD, Gilchrist MJ, Turner JM (2012) Rsx is a metatherian RNA with Xist-like properties in X-chromosome inactivation. Nature 487(7406):254 258. https://doi.org/10.1038/nature11171

Graves JA (2015) Weird mammals provide insights into the evolution of mammalian sex chromosomes and dosage compensation. J Genet 94(4):567-574

Johansson AM, Stenberg P, Bernhardsson C, Larsson J (2007) Painting of fourth and chromosomewide regulation of the 4th chromosome in Drosophila melanogaster. EMBO J 26(9):2307-2316. https://doi.org/10.1038/sj.emboj.7601604

Kaiser VB, Bachtrog D (2010) Evolution of sex chromosomes in insects. Annu Rev Genet 44:91-112. https://doi.org/10.1146/annurev-genet-102209-163600

Marshall Graves JA (2008) Weird animal genomes and the evolution of vertebrate sex and sex chromosomes. Annu Rev Genet 42:565-586. https://doi.org/10.1146/annurev.genet.42.110807.091714

Mulugeta E, Wassenaar E, Sleddens-Linkels E, van IJcken WFJ, Heard E, Grootegoed JA, Just W, Gribnau J, Baarends WM (2016) Genomes of Ellobius species provide insight into the evolutionary dynamics of mammalian sex chromosomes. Genome Res 26(9):1202-1210. https://doi.org/10.1101/ gr.201665.115

Pullirsch D, Hartel R, Kishimoto H, Leeb M, Steiner G, Wutz A (2010) The Trithorax group protein Ash2l and Saf-A are recruited to the inactive $\mathrm{X}$ chromosome at the onset of stable $\mathrm{X}$ inactivation. Development 137(6):935-943. https://doi.org/10.1242/dev.035956

Ruiz MF, Esteban MR, Donoro C, Goday C, Sanchez L (2000) Evolution of dosage compensation in Diptera: the gene maleless implements dosage compensation in Drosophila (Brachycera suborder) but its homolog in Sciara (Nematocera suborder) appears to play no role in dosage compensation. Genetics 156(4):1853-1865

Stenberg P, Larsson J (2011) Buffering and the evolution of chromosome-wide gene regulation. Chromosoma 120(3):213-225. https://doi.org/10.1007/s00412-011-0319-8 
Veyrunes F, Waters PD, Miethke P, Rens W, McMillan D, Alsop AE, Grutzner F, Deakin JE, Whittington CM, Schatzkamer K, Kremitzki CL, Graves T, Ferguson-Smith MA, Warren W, Marshall Graves JA (2008) Bird-like sex chromosomes of platypus imply recent origin of mammal sex chromosomes. Genome Res 18(6):965-973. https://doi.org/10.1101/gr.7101908

Veyrunes F, Chevret P, Catalan J, Castiglia R, Watson J, Dobigny G, Robinson TJ, Britton-Davidian J (2010) A novel sex determination system in a close relative of the house mouse. Proc Biol Sci 277(1684):1049-1056. https://doi.org/10.1098/rspb.2009.1925

Vicoso B, Bachtrog D (2013) Reversal of an ancient sex chromosome to an autosome in Drosophila. Nature 499(7458):332-335. https://doi.org/10.1038/nature12235

Vyskot B, Hobza R (2015) The genomics of plant sex chromosomes. Plant Sci 236:126-135. https://doi. org/10.1016/j.plantsci.2015.03.019

Wang X, Douglas KC, Vandeberg JL, Clark AG, Samollow PB (2014) Chromosome-wide profiling of $\mathrm{X}$-chromosome inactivation and epigenetic states in fetal brain and placenta of the opossum, Monodelphis domestica. Genome Res 24(1):70-83. https://doi.org/10.1101/gr.161919.113

Zhang Y, Malone JH, Powell SK, Periwal V, Spana E, Macalpine DM, Oliver B (2010) Expression in aneuploid Drosophila S2 cells. PLoS Biol 8(2):e1000320. https://doi.org/10.1371/journal. pbio. 1000320

Open Access This chapter is licensed under the terms of the Creative Commons Attribution 4.0 International License (http://creativecommons.org/licenses/by/4.0/), which permits use, sharing, adaptation, distribution and reproduction in any medium or format, as long as you give appropriate credit to the original author(s) and the source, provide a link to the Creative Commons license and indicate if changes were made.

The images or other third party material in this chapter are included in the chapter's Creative Commons license, unless indicated otherwise in a credit line to the material. If material is not included in the chapter's Creative Commons license and your intended use is not permitted by statutory regulation or exceeds the permitted use, you will need to obtain permission directly from the copyright holder.

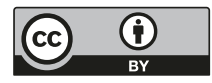

